\title{
Is robotic pancreatic surgery finally ready for prime-time?
}

\author{
Andrew A. Gumbs ${ }^{1}$, Roland Croner ${ }^{2}$, Elie Chouillard ${ }^{1}$ \\ ${ }^{1}$ Centre Hospitalier Intercommunal de Poissy/Saint-Germain-En-Laye, Poissy, France; ${ }^{2}$ Department of General-, Visceral-, Vascular- and \\ Transplantation Surgery, University Hospital Magdeburg, Magdeburg, Germany \\ Correspondence to: Andrew A. Gumbs, MD, FACS. Département de Chirurgie Viscérale et Digestive Centre Hospitalier de Poissy-Saint Germain, 10 \\ rue du Champ Gaillard, 78300 Poissy, France. Email: aagumbs@gmail.com. \\ Comment on: Liu R, Wakabayashi G, Palanivelu C, et al. International consensus statement on robotic pancreatic surgery. Hepatobiliary Surg Nutr \\ 2019;8:345-60.
}

Submitted Nov 28, 2019. Accepted for publication Dec 15, 2019.

doi: 10.21037/hbsn.2019.12.05

View this article at: http://dx.doi.org/10.21037/hbsn.2019.12.05

The white paper entitled, "International consensus statement on robotic pancreatic surgery", has been devised by leading minimally invasive surgeons to evaluate the current state for robotic pancreatic surgery. Six experts started the process by developing guidelines of robotic pancreatic surgery. Six experts started the process by developing guidelines of robotic pancreatic surgery. The guidelines were refined over a total of 4 meetings with the input and assistance of 20 additional international experts. A total of 19 recommendations involving the burgeoning field of robotic pancreatic surgery have been published in this article. With the recent publication of the International Summit on Laparoscopic Pancreatic Resection (ISLPR) "Coimbatore Summit Statements", and the LEOPARD- 1 and 2 trials this paper is particularly timely (1-3).

Few would argue that minimally invasive surgery is not the current gold standard for distal pancreatectomy. The LEOPARD-1 trial is a randomized, multi-center, and patient-blinded study from the Netherlands comparing laparoscopic to open distal pancreatectomy. Although complication rates were similar, the minimally invasive arm had improvements in functional recovery time and fewer problems with delayed gastric emptying. Unfortunately, the LEOPARD-2 trial did not see any advantages to laparoscopic pancreatoduodenectomy and the trial was closed after only 20 patients due to a $15 \%$ mortality rate in the minimally invasive arm. One must wonder if the first trial comparing laparoscopic and open pancreatoduodenectomy should have been done in a country that is not generally known to be an early adopter of this technique, especially when one considers the fact that some surgeons in France, the USA and India have been doing minimally invasive duodenopancreatectomy since the 1990's (4-6).

Several laparoscopic pancreatic surgeons recently published a white paper of their own specifically looking at the question of how to broaden the adoption of minimally invasive techniques to lesions in the head of the pancreas (3). Professor Palanivelu co-authored both of these white papers and has published a randomized control trial of open versus laparoscopic pancreatoduodenectomy in the British fournal of Surgery and mortality rates were identical. However, the minimally invasive group had a shorter hospital stay, a finding in direct contrast to the results of the LEOPARD-2 trial (7). Because of this dichotomy of results, 9 of the 19 recommendations in this consensus paper seemed particularly interesting to us, but raised a few questions.

"(Recommendation 3: there is no significant diffierence concerning the spleen-preserving rate between RDP and LDP; however, RDP has a higher splenic vessel-preserving rate, which renders RDP more suitable for spleenpreserving DP with splenic vessel preservation)". RDP = robotic distal pancreatectomy; LDP = laparoscopic distal pancreatectomy.

Much has been written about the superior rate of splenic vessel preservation with robotic distal pancreatectomy. If the rate of splenic preservation is similar when compared to laparoscopic distal pancreatectomy, then the recommendation to use robotics when spleen preserving distal pancreatectomy is indicated is confusing. Perhaps this approach has the potential to improve robotic duodenopancreatectomy, mainly through, the dissection of the uncinate process off of the portomesenteric confluence. 
Once this expertise has been mastered, however, the benefit of robotics to the actual patient undergoing the distal pancreatectomy seems questionable.

"Recommendation 6: there is insufficient evidence to support the view that the cost of RDP is higher than that of LDP or ODP; the cost-effiectiveness of RDP should be synthetically evaluated based on several factors, including overall healthcare expense, patient's psychological benefit, and social benefit".

Although this may be true after the initial cost of the robot is recouped or shifted to other specialities, is seems difficult to understand how the first 50 robotic pancreatic procedures with a robot dedicated to this type of surgery could compare economically to a pancreatic department that chooses to go the laparoscopic route. Factoring in all the upfront expenses to starting a laparoscopic program, laparoscopic equipment costs a fraction of the complete surgical system. Was the initial cost to purchase the robot accounted for when making this recommendation?

"Recommendation 7: for malignant tumors, robotic pancreaticoduodenectomy (RPD) is associated with higher $\mathrm{R} 0$ resection rate but similar lymph node harvest number compared to that with OPD".

In general, smaller tumors are approached minimally invasively when compared to open approaches, as a result, any comment on R-status may be problematic. Was tumor size factored in during the creation of this recommendation (8)?

"Recommendation 10: RPD has comparable perioperative mortality, overall postoperative complication rate, and the rate of POPF as that with OPD; however, RPD has shorter LOS than that with OPD".

This is an interesting finding, however, were tumor size and proximity to the great vessels accounted for during the development of this recommendation (please see discussion regarding Recommendation 7)?

"Recommendation 11: for a surgeon with extensive experience in laparoscopic pancreatectomy, the operative time will decrease significantly after 40 consecutive cases of RPD".

The question of how many procedures are needed for the learning curve is well discussed in this recommendation. However, as mentioned in the paper many robotic surgeons already have laparoscopic pancreatic surgical experience prior to embarking on adoption of the robot. If so, this could influence a comparison of robotic and laparoscopic outcomes. Do the authors advocate laparoscopic skills, if so how many laparoscopic procedures should be done prior to embarking on robotic approaches. Some robotic pancreatic surgeons do not have experience with laparoscopic pancreatic surgery and switch to laparoscopy once they have mastered robotics because of time that may be saved by using laparoscopy alone. Conversely, some laparoscopic surgeons switch to robotics because they feel more comfortable using the robot and prefer the increased degree of freedom with the EndoWrist once they try it. One wonders if robotics and laparoscopy should be considered as 2 separate techniques or are they simply 2 diffierent tool sets in the overall armamentarium of minimally invasive surgery?

"Recommendation 12: hybrid technique (laparoscopic/ robotic) can be used in $\mathrm{PD}$; for surgeons with extensive experience in laparoscopic surgery, a hybrid method can be utilized during the transition to total RPD".

Although the authors describe a hybrid technique where the resection is done laparoscopically with the reconstruction done robotically, it seems that the robot may be particularly beneficial during the ablative part of a duodenopancreatectomy. In particular, the third arm seems ideal to assist the surgeon in case of intra-operative bleeding and tissue retraction during complex dissection. This is highlighted by the finding that the rate of splenic vessel preservation is augmented during robotic distal pancreatectomy when compared to standard laparoscopy (see discussion regarding Recommendation 3). Although a complete robotic system is touted as being more "intuitive" and easier for open surgeons to adopt minimally invasive techniques, it seems from the authors own description that many surgeons start with laparoscopy and graduate to robotics after an extensive laparoscopic training period.

This is particularly true for duodenopancreatectomy. Perhaps the real value of the robot is not in converting open surgeons to minimally invasive surgeons, but lies in optimizing the ablative phase when complex vascular dissection and repair might be required. The acknowledgment that surgeons should attempt robotic pancreatic duodenopancreatectomy only after the learning curve of laparoscopic duodenopancreatectomy is overcome might get more pancreatic surgeons to adopt robotics.

"Recommendation 14: robotic central pancreatectomy is safe and feasible for benign and borderline tumors in the neck and proximal body of the pancreas. The distal pancreatic stump of at least $5 \mathrm{~cm}$ should be retained as suggested".

The first author of this consensus paper has described a central pancreatectomy with a primary anastomosis of the 2 cut pancreatic ends (9). Due to the high rate of complications with standard robotic central pancreatectomy discussed in this paper, it seems LIKELY that primary pancreatic anastomosis might be preferable during robotic central pancreatectomy. 
"Recommendation 15: there is insufficient evidence to support the view that short-term outcomes of robotic central pancreatectomy are better than that of open central pancreatectomy".

This statement seems a bit confusing. According to the increased length of stay, operative time, blood loss, delayed gastric emptying and fistula rate after robotic central pancreatectomy when compared to open pancreatectomy, robotic central pancreatectomy seems to not be currently indicated. Do the authors have any ideas as to why this might be the case? It seems that the authors found improved results after robotic duodenopancreatectomy, where a pancreatic anastomosis is fashioned. Do robotic surgeons preferentially do a pancreaticogastrostomy or do they use the jejunum for the anastomosis? Perhaps the above mentioned primary pancreatic anastomosis after central pancreatectomy should be advocated during robotic approaches (see discussion of Recommendation 14).

"Recommendation 19: the surgical concept of RPD is diffierent from that of OPD due to diffierent view angles during surgery, lack of tactile feedback, and more dependence on the operative instruments in robotic surgery".

In this section, the authors state that, "...the suturing ability in RPD was better than that in LPD, but still much less straightforward than that in OPD". The authors appropriately state that haptics are a problem during robotic surgery, but then state that suturing is superior when compared to laparoscopy, which does have haptics because the EndoWrist of the robot has increased degrees of freedom at its tip. Many laparoscopic pancreatic surgeons resist adopting the complete surgical system because of the absence of haptics, in particular, due to concerns of encountering a soft pancreas. A pancreatic anastomosis of a soft pancreas can be an extremely challenging endeavor even during an open procedure. What do the authors do when confronted with a soft pancreas and a small pancreatic duct? To maintain haptics in roboticallyassisted surgery, some authors have suggested that smaller robotic devices such as hand-held robotic instruments may be a way to have the benefits of robotics while at the same time maintaining a sense of touch (5).

We applaud the authors on an excellent consensus paper that is both timely and well-researched. We look forward to following the authors future research in the growing and exciting field of minimally invasive robotic pancreatic surgery.

\section{Acknowledgments}

Funding: None.

\section{Footnote}

Provenance and Peer Review: This article was commissioned by the editorial office, Hepatobiliary Surgery and Nutrition. The article did not undergo external peer review.

Conflicts of Interest: All authors have completed the ICMJE uniform disclosure form (available at http://dx.doi. org/10.21037/hbsn.2019.12.05). The authors have no conflicts of interest to declare.

Ethical Statement: The authors are accountable for all aspects of the work in ensuring that questions related to the accuracy or integrity of any part of the work are appropriately investigated and resolved.

Open Access Statement: This is an Open Access article distributed in accordance with the Creative Commons Attribution-NonCommercial-NoDerivs 4.0 International License (CC BY-NC-ND 4.0), which permits the noncommercial replication and distribution of the article with the strict proviso that no changes or edits are made and the original work is properly cited (including links to both the formal publication through the relevant DOI and the license). See: https://creativecommons.org/licenses/by-nc-nd/4.0/.

\section{References}

1. de Rooij T, van Hilst J, van Santvoort H, et al. Minimally Invasive Versus Open Distal Pancreatectomy (LEOPARD): A Multicenter Patient-blinded Randomized Controlled Trial. Ann Surg 2019;269:2-9.

2. van Hilst J, de Rooij T, Bosscha K, et al. Laparoscopic versus open pancreatoduodenectomy for pancreatic or periampullary tumours (LEOPARD-2): a multicentre, patient-blinded, randomised controlled phase $2 / 3$ trial. Lancet Gastroenterol Hepatol 2019;4:199-207.

3. Palanivelu C, Takaori K, Abu Hilal M, et al. International Summit on Laparoscopic Pancreatic Resection (ISLPR) "Coimbatore Summit Statements". Surg Oncol 2018;27:A10-5.

4. Gagner M, Pomp A. Laparoscopic pylorus-preserving pancreatoduodenectomy. Surg Endosc 1994;8:408-10.

5. Gumbs AA, Croner R, Rodriguez A, et al. 200 consecutive laparoscopic pancreatic resections performed with a robotically controlled laparoscope holder. Surg Endosc 2013;27:3781-91.

6. Palanivelu C, Jani K, Senthilnathan P, et al. Laparoscopic pancreaticoduodenectomy: technique and outcomes. J Am 
Coll Surg 2007;205:222-30.

7. Palanivelu C, Senthilnathan P, Sabnis SC, et al. Randomized clinical trial of laparoscopic versus open pancreatoduodenectomy for periampullary tumours. Br J Surg 2017;104:1443-50.

8. Zhang H, Lan X, Peng B, et al. Is total laparoscopic

Cite this article as: Gumbs AA, Croner R, Chouillard E. Is robotic pancreatic surgery finally ready for prime-time? HepatoBiliary Surg Nutr 2020;9(5):650-653. doi: 10.21037/ hbsn.2019.12.05 pancreaticoduodenectomy superior to open procedure? A meta-analysis. World J Gastroenterol 2019;25:5711-31.

9. Wang ZZ, Zhao GD, Zhao ZM, et al. An end-to-end pancreatic anastomosis in robotic central pancreatectomy. World J Surg Oncol 2019;17:67. 